Original article

\title{
Genetic improvement of laying hens viability using survival analysis
}

\author{
Vincent Ducrocq ${ }^{\mathrm{a}, *}$, Badi Besbes ${ }^{\mathrm{b}}$, Michel Protais ${ }^{\mathrm{c}}$ \\ ${ }^{a}$ Institut national de la recherche agronomique, Station de génétique quantitative \\ et appliquée, 78352 Jouy-en-Josas Cedex, France \\ b Hubbard-ISA Centre de Sélection, BP 27, 35220 Chateaubourg, France \\ ${ }^{\mathrm{c}}$ Hubbard-ISA, Le Foeil, 22800 Quintin, France
}

(Received 26 May 1999; accepted 22 November 1999)

\begin{abstract}
The survival of about eight generations of a large strain of laying hens was analysed separating the rearing period (RP) from the production period ( $\mathrm{PP})$, after hens were housed. For RP (respectively PP), 97.8\% (resp., 94.1\%) of the 109160 (resp., 100665 ) female records were censored after 106 days (resp., 313 days) on the average. A Cox proportional hazards model stratified by flock (= season) and including a hatch-within-flock (HWF) fixed effect seemed to reasonably fit the RP data. For PP, this model could be further simplified to a non-stratified Weibull model. The extension of these models to sire-dam frailty (mixed) models permitted the estimation of the sire genetic variances at $0.261 \pm 0.026$ and $0.088 \pm 0.010$ for RP and PP, respectively. Heritabilities on the log scale were equal to 0.48 and 0.19 . Nonadditive genetic effects could not be detected. Selection was simulated by evaluating all sires and dams, after excluding all records from the last generation. Then, actual parents of this last generation were distributed into four groups according to their own pedigree index. Raw survivor curves of the progeny of extreme parental groups substantially differed (e.g., by $1.7 \%$ at 300 days for PP), suggesting that selection based on solutions from the frailty models could be efficient, despite the very large proportion of censored records.
\end{abstract}

survival analysis / viability / laying hens / selection

Résumé - Amélioration génétique de la viabilité des poules pondeuses à partir d'une analyse de survie. Les données de survie d'environ huit générations d'une souche de grande taille de poules pondeuses ont été analysées en séparant la période d'élevage $(\mathrm{PE})$ de la période de production $(\mathrm{PP})$ après la mise en cage des poules. Pour PE (respectivement PP), 97,8 \% (resp., 94,1 \%) des 109160 (resp., 100665 ) performances femelles étaient censurées, après en moyenne 106 jours (resp., 313 jours). Un modèle à risques proportionnels de Cox stratifié par cheptel et incluant un effet fixé du lot de naissance intra cheptel semble décrire raisonnablement bien les données de la PE. Pour la PP, ce modèle peut être encore simplifié en un modèle de Weibull non stratifié. En étendant ces modèles à des modèles de fragilité (modèles mixtes) pèremère, les variances génétiques «pères » ont été estimées à 0,261 $\pm 0,026$ et 0,088 \pm

* Correspondence and reprints

E-mail: ducrocq@dga.jouy.inra.fr 
0,010 pour PE et PP respectivement (soit des héritabilités sur l'échelle logarithmique de 0,48 et 0,19 ). Il n'a pas été possible de détecter des effets génétiques non additifs. Une sélection a été simulée en évaluant tous les animaux parents, après avoir exclu les enregistrements de la dernière génération. Ensuite, les parents de cette dernière génération ont été répartis en quatre groupes suivant leur propre valeur génétique sur ascendance. Les courbes de survie brutes des descendants des groupes parentaux extrêmes diffèrent substantiellement (par exemple, de 1,7\% à 300 jours pour PP). Ceci suggère clairement qu'une sélection basée sur les solutions des modèles de fragilité pourrait être efficace, malgré la proportion très élevée de données censurées.

analyse de survie / viabilité / poules pondeuses / sélection

\section{INTRODUCTION}

For any domestic species, mortality rate is an important trait which must be kept at a minimum. Mortality has obvious economic consequences: dead animals are worthless, they increase replacement costs and decrease overall performance, expressed per animal born or kept in production. From a welfare point of view, even low mortality rates should be reduced further, as they may reflect an inadequacy of the production system.

In poultry, mortality rates are generally low. Under controlled conditions, average mortality is usually below $5.2 \%$ per year of egg production [21]. This rate tends to increase [1]. Selection for lower mortality has been practised directly or indirectly for many years, but it has not been very effective [21]. Heritability estimates for mortality of pure-line hens in single cages are typically near zero, because the level of mortality is too low to express significant family differences [13]. Improvement of such a trait with low incidence is a formidable challenge to the geneticist.

The main characteristic of survival analysis is that it uses all the information available, from dead animals as well as from animals still alive when the analysis is performed (resulting in so-called censored records). It describes at what rate animals are dying over time. This is in contrast with other techniques describing survival at a given point in time, as a $0 / 1$ trait. Survival analysis is becoming a standard technique for the genetic analysis of length of productive life of some species (e.g., in dairy cattle [7, 9-11]).

The aims of this study are to find a proper model for the genetic analysis of survival data of a large strain of laying hens and to assess its potential use in selection programs.

\section{MATERIAL AND METHODS}

\subsection{Breeding structure}

This study was based on survival information collected on a total of 130442 birds from a commercial laying strain under selection at "Hubbard-ISA". Only female survival is considered here, representing 109160 laying hens. These hens were raised in 17 successive flocks, each one consisting of 4 to 8 hatches. Parents of the first flocks were also added. Hence, in total, the pedigree file included animals from 20 flocks, representing about eight generations. 
The total number of parents was 1121 males and 6479 females. On the average, each male was mated to 6.8 females (range: 1 to 17 ) and had 97.4 female progeny (range: 1 to 193). Each female parent had an average of 16.8 female progeny (range: 1 to 58 ).

The inbreeding coefficient of each individual in the initial data set was computed with all the information available, that is assuming that animals of the initial flocks ( 1 to 3 ) were unrelated. As a consequence, virtually no animal born in flock 1 to 9 was inbred. Later, the inbreeding coefficient increased at an approximately linear rate of $+0.26 \%$ per flock $(+0.61 \%$ per generation $)$ and reached $2.6 \pm 0.8 \%$ in flock 20 for both sexes. Inbreeding was ignored in all analyses, given its modest value and its homogeneity within a flock.

\subsection{Material}

For each hen, the initial data set included her date of birth, date when housed and date of death or removal. Records of removed live animals were considered as censored. Figure 1 displays the Kaplan-Meier estimate of the (raw) survivor curve [18] of all animals, considering birth as the initial point. The curve is smooth but presents a change of slope at about 100 days, which coincides with the time when the hens were housed in individual cages. A precise analysis of the data clearly showed a different mortality rate during the two periods separated by this event: the rearing period (RP) and the production period $(\mathrm{PP})$. Therefore, two new longevity measures were defined: length of rearing life (LRL) where birth is considered as the initial point and animals still alive when housed are considered as censored; and length of productive life (LPL) starting when the hen was housed in an individual cage. LPL records of animals removed before death were considered as censored. Figure 1 also shows that

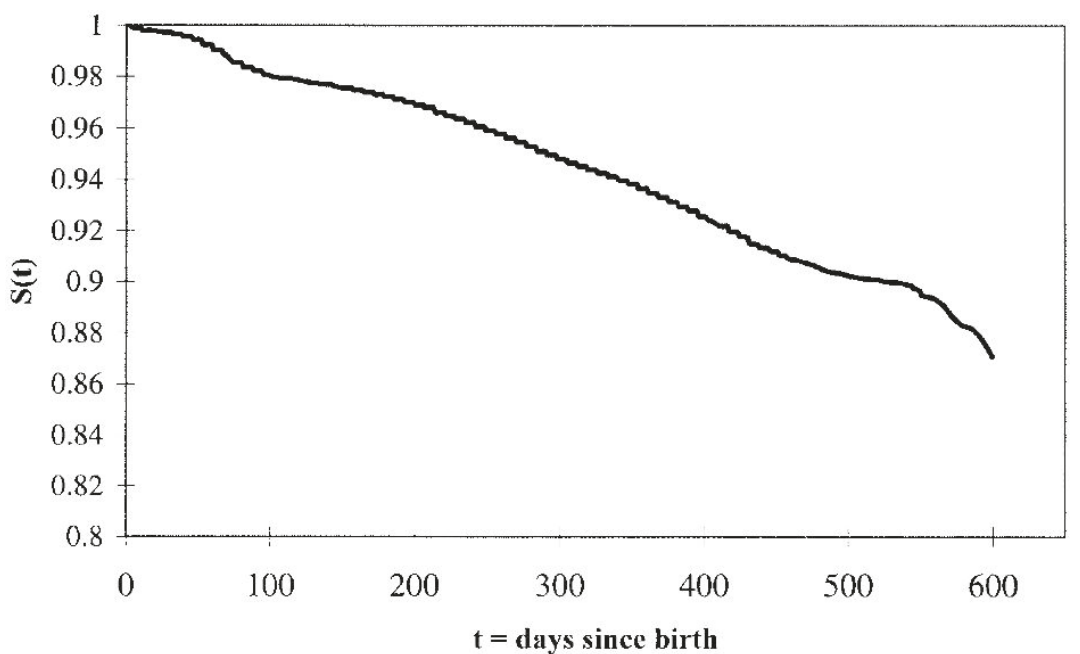

Figure 1. Kaplan-Meier non-parametric estimate of the survival curve of the whole population. 
after about 500 days, the raw survivor curve was not as smooth as before. In fact, most hatches had been terminated then and few animals (or hatches) were still at risk. Therefore, it was decided to ignore the late period of LPL (after 400 days of production), by considering all records of animals still alive then as censored at $\mathrm{T}=400$ days.

\subsection{Models}

\section{Theor}

A popular model for the analysis of survival data is the proportional hazards model $[3,17,20]$ for which the hazard function $h\left(t ; \mathbf{x}_{m}\right)$ at time $t$ of a particular animal $m$, characterised by a set of explanatory variables $\mathbf{x}_{\mathbf{m}}$, is written as:

$$
h\left(t ; \mathbf{x}_{m}\right)=h_{0}(t) \exp \left\{\mathbf{x}_{m}^{\prime} \beta\right\}
$$

where $h_{0}($.$) is called the baseline hazard function and \beta$ is a vector of regression parameters. $h_{0}($.$) is either completely arbitrary (in the so-called 'Cox model') or$ may have a known parametric form. One of the most frequently used parametric forms is the Weibull hazard function $\left(h_{0}(t)=\lambda \rho(\lambda t)^{\rho-1}\right.$, where $\rho$ and $\lambda$ are two positive parameters). The Weibull survivor function $\left(S(t)=\exp \left\{-(\lambda t)^{\rho}\right\}\right)$ is a generalisation of the exponential survivor function, for which $\rho=1$. The resulting model (1) is a Weibull regression model. The term 'proportional hazards model' comes from the fact that the ratio of the hazards of two animals $m$ and $m^{\prime}$

$$
\frac{h\left(t ; \mathbf{x}_{m}\right)}{h\left(t ; \mathbf{x}_{m^{\prime}}\right)}=\exp \left\{\left(\mathbf{x}_{m}-\mathbf{x}_{m^{\prime}}\right)^{\prime} \beta\right\}
$$

is constant over time.

If this initial model (1) is considered too restrictive for a good fit of the data, it can be extended in several ways. The simplest one consists in defining a different baseline hazard function $h_{0, n}($.$) for each level (or stratum) n$ of a particular factor. Again, these baseline hazard functions can be either arbitrary (stratified Cox model) or Weibull (stratified Weibull regression model). A careful examination of the baselines in a stratified Cox model is a way to check the proportional hazards assumption or the validity of a Weibull model: from each hazard function, it is possible to estimate a baseline survivor function $\hat{S}_{0, n}$ and if a Weibull model is adequate, a plot of $\log \left(-\log \hat{S}_{0, n}\right)$ vs. $\log t$ should give a straight line. Furthermore, if these lines computed for different strata are parallel, the proportional hazards assumption holds and a unique baseline can be used over strata [17].

For completeness, it must be said that other generalisations of model (1) exist. In particular, $\mathbf{x}_{m}$ can be described as a function $\mathbf{x}_{m}(t)$ of time $t$. If $\mathbf{x}_{m}(t)$ is piecewise constant, the proportional hazards assumption must hold only within intervals for which $\mathbf{x}_{m}(t)$ and $\mathbf{x}_{m^{\prime}}(t)$ are constant, and no longer on the whole time scale, from $t=0$ to $+\infty$. The inclusion of time dependent covariates was not needed here.

A final extension of proportional hazards models is the addition of random effects. The resulting mixed models are called 'frailty models' in statistics [22]. 
We write:

$$
h\left(t ; \mathbf{x}_{m}, \mathbf{z}_{m}\right)=h_{0}(t) \exp \left\{\mathbf{x}_{m}^{\prime} \beta+\mathbf{z}_{m}^{\prime} \mathbf{u}\right\}
$$

where $\mathbf{u}$ is a vector of (possibly correlated) random variables with associated incidence vector $\mathbf{z}_{m}$. A detailed description of mixed survival models can be found in [8].

\subsection{Model selection}

A preliminary analysis was conducted to decide whether the proportional hazards assumption was valid across flocks and hatches. Because a general Cox model is computationally more demanding, it was also checked whether a simplified parametric form could be retained for the baseline(s). Both for the LRL and the LPL of an animal $m$, the following initial stratified Cox model was used:

$$
h(t ; m)=h_{0, n}(t) \exp \left\{b_{k}\right\}
$$

where $b_{k}$ represented the $k$ th hatch within flock $(\mathrm{HWF})$ fixed effect $(k=1$ to 130$)$. The baseline $h_{0, n}($.$) was initially stratified by hatch within a flock$ (i.e., $n=k)$. Values of $\log \left(-\log \hat{S}_{0, n}(t)\right)$ were plotted against $\log t$ to decide whether the baselines could be approximated with a Weibull baseline hazard function. Furthermore, whenever the plot displayed parallel lines over strata, these were grouped together by flock. Then, if parallel lines were obtained again, stratification was ignored. All these tests are simple graphical tests. They were preferred to formal tests because it was considered that the assumptions tested remain approximations of the correct model (see p. 354 in [20]). The purpose of these graphical tests is essentially to avoid substantial discrepancies which could invalidate further inferences.

When a final model was chosen for the fixed effects part, the estimated generalised residual [4] was computed for each animal $m$ dying or censored at time $y_{m}$ as:

$$
\hat{e}_{m}=\int_{0}^{y_{m}} \hat{h}_{0, n}(t) \exp \left\{\hat{b}_{k}\right\} \mathrm{d} t
$$

If the proportional hazards model is correct, the true values of these generalised residuals should follow a (censored) unit exponential distribution [5]. Hence, a plot of the sorted $\hat{e}_{m}$ 's against the expected order statistics of a censored unit exponential distribution should display a straight line with slope 1 and going through the origin.

\subsection{Genetic model}

To account for genetic effects, the previously selected fixed effects models for LRL and LPL were extended to mixed models as:

$$
h(t ; m)=h_{0, n}(t) \exp \left\{b_{k}+s_{i}+d_{j}\right\}
$$

where $i$ and $j$ are the sire and the dam of animal $m$ and $s_{i}$ and $d_{j}$ the corresponding sire and dam effects. If these effects are grouped together into a 
vector $\mathbf{g}$ of genetic effects, and if only additive genetic effects influence longevity, then under polygenic inheritance $\mathbf{g}$ follows a multivariate normal distribution:

$$
\mathbf{g} \sim \operatorname{MVN}\left(\mathbf{0}, \mathbf{A} \sigma_{g}^{2}\right)
$$

where $\mathbf{A}$ is the additive genetic relationship between all male and female parents.

However, non-additive effects may exist, biasing the estimation of $\sigma_{g}^{2}[23]$. Such effects are likely to mainly influence covariances between full-sibs. To get unbiased additive genetic effects and to assess the importance of non-additive effects, the following model was used:

$$
h(t ; m)=h_{0, n}(t) \exp \left\{b_{k}+s_{i}+d_{j}+c_{i j}\right\}
$$

where $c_{i j}$ represents a full-sib effect, characteristic of progeny of the mating pair $(i, j)$. For technical reasons, these full-sib effects were assumed to be iid log-gamma distributed with a mean equal to 1 , that is depending on a unique parameter $\gamma$. The choice of a log-gamma distribution for random effects in mixed survival models (or, equivalently, of a gamma distribution for the frailty term $\exp \left\{c_{i j}\right\}$ ) is usual in frailty models, because of their flexibility and mathematical convenience $[2,6,10,19,20]$. A normal distribution for $c_{i j}$ would have been probably more intuitive in an animal breeding context, but it must be noted that when $\gamma$ becomes large, the log-gamma distribution tends to a normal distribution $[10,17]$ and the two alternatives are then similar. The variance of the full-sib effect is equal to $\Psi^{(1)}(\gamma)$, where $\Psi^{(1)}($.$) is the trigamma$ function. For large $\gamma^{\prime}$ 's, this variance is approximately equal to $\gamma^{-1}$.

The sire (or dam) variance $\sigma_{g}^{2}$ as well as the $\gamma$ parameter of the log-gamma distribution of $c_{i j}$ were estimated using the Bayesian approach described in [8]. Non-informative priors were used for the fixed effects and the dispersion parameters $\sigma_{g}^{2}$ and $\gamma$. Multivariate normal (for $\mathbf{g}$ ) and $\log$-gamma (for $c_{i j}$ ) priors were combined with the likelihood function of the data to obtain an expression proportional to the joint posterior density of all parameters. Whenever a Cox model was used, the likelihood function was replaced by a partial likelihood [3] which does not contain any information about the arbitrary baseline hazard function. The marginal posterior density of $\sigma_{g}^{2}$ and $\gamma$ was obtained by integrating out all the other parameters. The integration was algebraic and therefore exact in the case of the log-gamma effects $c_{i j}$ 's but was performed using a Laplace approximation for all other parameters. In fact, only the mode and the first three moments of $\sigma_{g}^{2}$ were computed. All technical details are given in [8]. Computations were done using "The Survival Kit-V3.0", a set of Fortran programs written with animal breeding applications in mind [12].

Estimated genetic effects $\hat{s}_{i}$ and $\hat{d}_{j}$ were computed assuming that the value of $\sigma_{g}^{2}$ at the mode of the marginal posterior distribution was the correct value. The mean values for each flock were used to estimate genetic trends.

\subsection{Simulated selection}

Given the relatively long period covered in the data set (about eight generations), it was possible to retrospectively simulate selection on LRL or 
LPL estimated breeding values. Let $\mathcal{P}$ represent the set of parents of the last (20th) flock and let $\mathcal{G} \mathcal{P}$ be the set of parents of animals in $\mathcal{P}$. The genetic evaluation of all sires and dams in the $\mathcal{G} \mathcal{P}$ group and their ancestors was based on all (possibly censored) longevity records available when the mating pairs were formed among $\mathcal{P}$. This evaluation was performed using the appropriate model and the genetic parameters obtained in the previous (global) analysis. Pedigree values $\hat{s}_{i}$ for sires and $\hat{d}_{j}$ for dams were calculated for each animal in set $\mathcal{P}$, averaging the estimated breeding values of their parents. The next step in a selection program for longevity only would involve the choice of the best males and the best females based on their pedigree values and the mating of the selected animals. Here, males and females were already selected according to a completely different criterion and they were mated irrespective of their (then unknown) pedigree value for longevity. It was however possible to approximate a selection step by sorting the animals in flock 20 according to the value of $0.5\left(\hat{s}_{i}+\hat{d}_{j}\right)$, i.e., their pedigree value based on grand-parental breeding values only. Using this approach, flock 20 was partitioned into 4 groups of equal size. Each group consisted of 1615 and 1576 individuals, respectively, for LRL and LPL. The Kaplan-Meier (raw) survivor curves were computed for each group and then compared. A difference between these survivor curves would be an indication on whether selection using the results of the survival analysis could be efficient.

In order to check whether these observed survivor curves correspond to the expected ones, the expected survivor curves for the extreme groups were computed based on a development similar to Foulley $[14,15]$ for the prediction of response to selection on discrete data analysed using nonlinear models. The formula for these survivor curves is briefly outlined here (for details on the derivation, see $[14,15])$.

Consider model (3) with only one normally distributed random effect $u$. Let $\mathcal{G}$ be a group of individuals selected based on their estimated breeding value $\hat{u}$. For example, assume that the individuals with the largest $25 \%$ estimated breeding values are retained, corresponding to a selection threshold $\tau$ on the distribution of $\hat{u}$. The value of the expected survivor curve at time $t$ of the individuals in $\mathcal{G}$ raised in a particular environment characterized by the hatch within flock effect $b_{k}$ is:

$$
\begin{aligned}
\mathcal{S}\left(t \mid \mathcal{G}, h_{0}(.), b_{k}\right) & =\int_{\tau}^{+\infty} S\left(t \mid h_{0}(.), b_{k}, \hat{u}\right) p(\hat{u}) \mathrm{d} \hat{u} \\
& =\int_{\tau}^{+\infty}\left[\int_{-\infty}^{+\infty} S\left(t \mid h_{0}(.), b_{k}, u\right) p(u \mid \hat{u}) \mathrm{d} u\right] p(\hat{u}) \mathrm{d} \hat{u}
\end{aligned}
$$

where $p(\hat{u})$ and $p(u \mid \hat{u})$ are normal density functions with mean of 0 and $\hat{u}$ and variance of $\kappa^{2} \sigma_{u}^{2}$ and $\left(1-\kappa^{2} \sigma_{u}^{2}\right)$, respectively, $\kappa^{2}$ being the accuracy of the evaluation of $u$.

This formula was adapted to model (8), with an extra level of integration for the full-sib effect $c_{i j}$ and replacing the effect $u$ by $s_{i}+d_{j}$. It was then applied to the extreme groups described above, using the estimated Weibull parameters for the baseline and the average estimated hatch within flock effect in the last flock for $b_{k}$ and replacing $\kappa^{2}$ by twice the average reliabilities of pedigree values 
for the animals in $\mathcal{P}$. The expected survivor curve for each group was finally compared to the actual (Kaplan-Meier) estimate of the survivor curve.

\section{RESULTS}

\subsection{General statistics}

Mortality rate among female chicks during the rearing period was about $2.2 \%$. In other words, $97.8 \%$ of LRL records were censored, at about 106 days on the average. Some animals were discarded before being put into individual cages. Among the 100665 remaining hens, 94.1\% of them were still alive when their flock was terminated, 313 days on the average after being housed.

\subsection{Choice of an adequate model}

Figure 1 shows the Kaplan-Meier estimate $\hat{S}_{K M}(t)$ of the survivor curve, over the whole period (rearing + production). A plot of $\log \left(-\log \hat{S}_{K M}(t)\right)$ against $\log t$ (Fig. 2) confirms that mortality rates were different before and after the moment when hens were put into individual cages. Before 105 days (i.e., $\log t \sim 4.65)$, a linear approximation of the curve gave a slope of $1.59\left(\mathrm{R}^{2}=\right.$ $0.98)$ while after that date, the slope was $1.14\left(\mathrm{R}^{2}=0.99\right)$. Therefore, it seems more adequate to analyse separately the rearing period and the production period. Note that the plot appears as a step function, especially during the rearing period, because mortalities were most often recorded on a weekly basis.

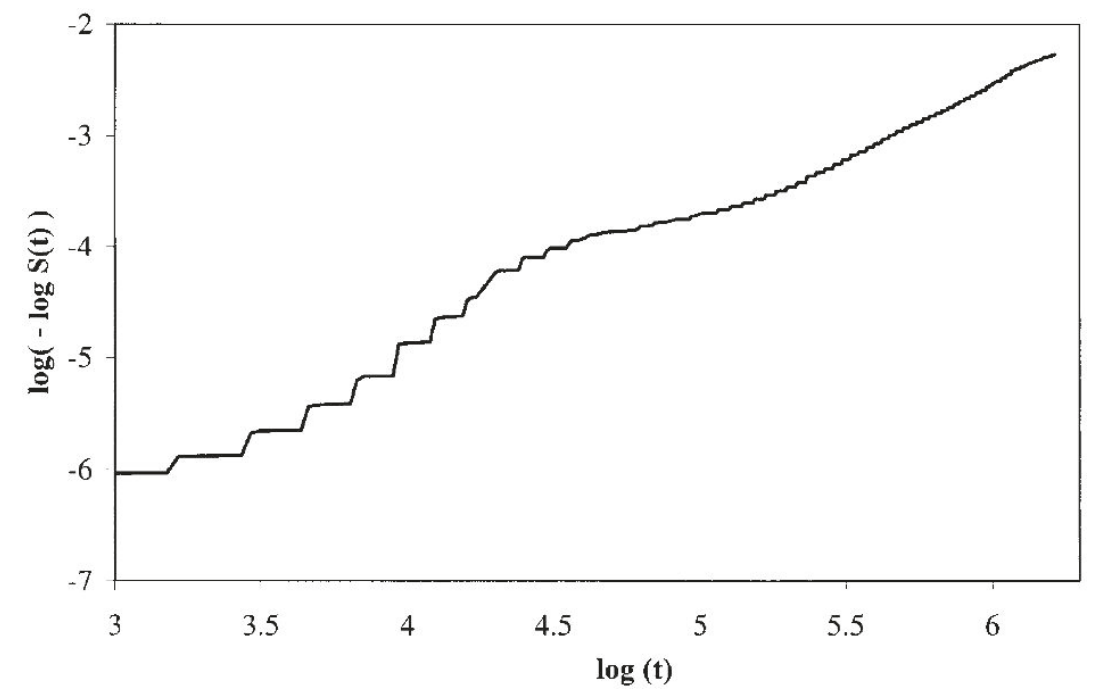

Figure 2. Graphical test of the assumption that the baseline hazard function for the whole population is a Weibull hazard ( $t=$ time expressed in days since birth).

Figure 2 suggests that for each period, the assumption of a Weibull baseline hazard function is plausible. To go further, the Kaplan-Meier estimates of 
the survivor curves for different strata were computed separately. Strata were defined as flocks or hatches within flocks. For all strata, $\log \left(-\log \hat{S}_{K M, n}(t)\right)$ was plotted against $\log t$, where $n$ is the index for stratum. For LPL, $t$ now refers to time since housed. Straight parallel lines would simultaneously indicate that the baseline hazard functions are Weibull hazards and that they are proportional, for any pair of strata. This was roughly what was observed for LPL, whether records were stratified by hatch within the flock (not shown) or simply by flocks (Fig. 3 - for clarity, only the last 12 flocks are represented). In contrast, for LRL, stratification by flock (Fig. 4) suggests that the baseline survivor functions vary across flocks and that for some flocks, the Weibull assumption is violated. Within a flock, the log-log transformation ofthe baselines for each hatch led to parallel lines. In other words, the appropriate models to analyse the two longevity measures seem to be a flock-stratified Cox model for LRL and a regular Weibull model for LPL.

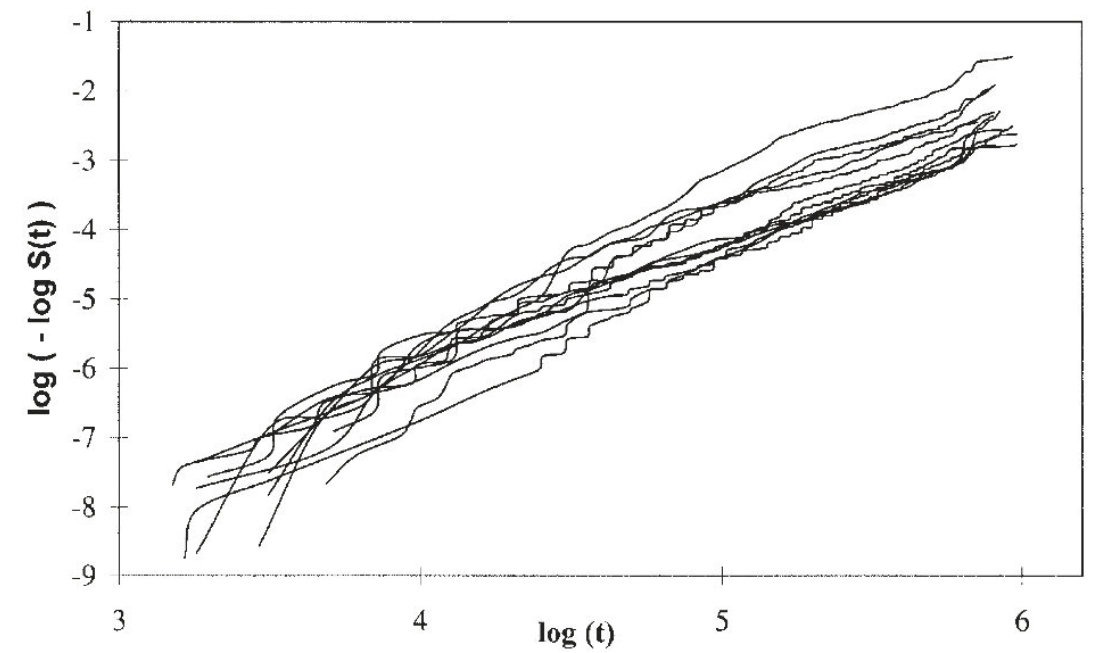

Figure 3. Graphical test of the assumption that the baseline hazard functions for survival during the production period and in different flocks are Weibull hazards ( $t=$ time since housed, last 12 flocks represented).

The overall goodness-of-fit check using the estimated generalised residuals $\hat{e}_{m}$ 's gave disappointing results: for example, a plot of the sorted residuals for LPL against the expected order statistics of a unit (censored) exponential distribution showed a straight line with slope 0.98 and intercept 0.0009 (when 1 and 0 were expected) and with an $\mathrm{R}^{2}$ of 0.997 ! Such results are likely to lead to the overoptimistic conclusion that the fit is perfect. The danger of such an erroneous inference was already indicated by Cox and Oakes (see p. 109 in [5]) and observed in some (but not all) situations in [6]: replacing the true generalised residuals $e_{m}$ by their computed values $\hat{e}_{m}$ ignores the fact that the $\hat{e}_{m}$ 's are not independent, leading to a "spuriously good fit" [5]. The very large fraction of censored records seems to make things even worse: virtually all $\hat{e}_{m}$ 's are very small. 


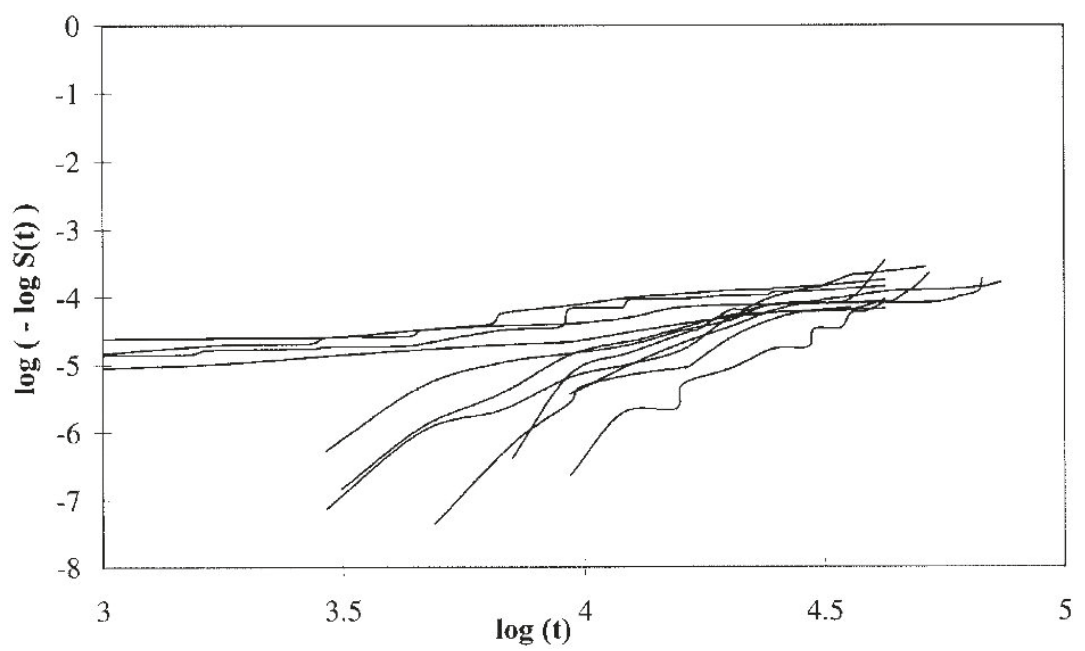

Figure 4. Graphical test of the assumption that the baseline hazard functions for survival during the rearing period and in different hatches are Weibull hazards ( $t=$ time since birth, 11 hatches from last 3 flocks represented).

\subsection{Variance component estimation}

The stratified Cox model for LRL and the Weibull model for LPL were extended to frailty models including sire and dam effects. Estimates of the sire (= dam) variance are reported in Table I. For both traits, the approximate posterior densities of the sire variance were only slightly skewed. As a consequence, the mode and the mean of these distributions were very close. A remarkable result is that despite the very high censoring rate, the standard deviations of the posterior densities were small: the size of the data set and the good pedigree structure allowed a precise estimation of sire variance. Its estimate for LPL was 0.088, which corresponds to a heritability on the log scale equal to [8]:

$$
h^{2}=\frac{4 \sigma_{g}^{2}}{\operatorname{Var}(\log T)}=\frac{4 \sigma_{g}^{2}}{\frac{\pi^{2}}{6}+2 * \sigma_{g}^{2}}=0.193 .
$$

For LRL, the sire variance was much larger. The residual variance on the transformed scale is still $\frac{\pi^{2}}{6}$ for the Cox model, leading to a heritability estimate of 0.482 .

These values should be interpreted with caution: they represent the heritability of the trait in the unrealistic ideal situation of no censoring. They are useful to compute approximate reliabilities of estimated breeding values using selection index theory, based on the actual number of uncensored observations [7].

As expected, the Weibull model was less computationally demanding than the stratified Cox model (about 30 times faster with the software used). 
Table I. Estimates of genetic parameters.

\begin{tabular}{|c|c|c|c|c|c|}
\hline \multirow{2}{*}{$\begin{array}{l}\text { Trait } \\
\text { Model }\end{array}$} & \multicolumn{2}{|l|}{$\mathrm{LRL}^{a}$} & \multicolumn{3}{|c|}{$\mathrm{LPL}^{a}$} \\
\hline & $\operatorname{Cox}^{b}$ & Weibull $^{c}$ & + full-sib $^{d}$ & Weibull $^{c}$ & + full-sib $^{d}$ \\
\hline \multicolumn{6}{|c|}{ Sire $(=$ dam $)$ variance $\sigma_{g}^{2}$ : } \\
\hline Mode $^{e}$ & 0.260 & 0.261 & 0.216 & 0.088 & 0.077 \\
\hline $\operatorname{Mean}^{e}$ & 0.261 & 0.262 & 0.218 & 0.089 & 0.078 \\
\hline Standard deviation ${ }^{e}$ & 0.026 & 0.026 & 0.024 & 0.010 & 0.010 \\
\hline Skewness coefficient ${ }^{e}$ & 0.219 & 0.248 & 0.252 & 0.225 & 0.266 \\
\hline \multicolumn{6}{|l|}{ Other parameters: } \\
\hline Heritability $^{f}$ & 0.482 & 0.482 & 0.390 & 0.194 & 0.167 \\
\hline $\begin{array}{l}\text { Weibull } \\
\text { parameter }(\rho)\end{array}$ & - & $\begin{array}{c}1.47 \pm \\
0.03\end{array}$ & $\begin{array}{c}1.48 \pm \\
0.03\end{array}$ & $\begin{array}{c}1.64 \pm \\
0.02\end{array}$ & $\begin{array}{c}1.64 \pm \\
0.02\end{array}$ \\
\hline$\gamma$ & - & - & $7.7^{g}$ & - & $23.1^{g}$ \\
\hline CPU time ${ }^{h}$ & $7 \mathrm{~h} 20 \mathrm{~m}$ & $13 \min 30$ & $14 \min 12 \mathrm{~s}$ & $9 \min 20 \mathrm{~s}$ & $10 \mathrm{~min} 20 \mathrm{~s}$ \\
\hline
\end{tabular}

${ }^{a}$ LRL (LPL): length of life during the rearing (productive) period; ${ }^{b}$ stratified by flock; ${ }^{c}$ not stratified; ${ }^{d}$ with a log-gamma full-sib effect; ${ }^{e}$ mode and first three moments of the approximate marginal posterior density of $\sigma_{g}^{2} ;{ }^{f}$ on the log scale; ${ }^{g} \gamma$ tends to $+\infty$ (zero variance) if if is not fixed. Here, an arbitrary value $\left(=0.5 \hat{\sigma}_{g}^{-2}\right)$ was imposed; ${ }^{h}$ indicative CPU time on an IBM Risc 595 AIX4.21.

This was mainly due to a much sparser Hessian matrix. To see whether the refinement in the model consisting of defining a different arbitrary baseline for each flock had an impact on the estimation of the sire variance, the same Weibull model as for LPL was fitted to LRL data. All the characteristics of the marginal posterior density of $\sigma_{g}^{2}$ were virtually unchanged. The heritability on the log scale was again estimated at 0.482 .

Model (6) assumes that the sire and dam effects are additive genetic effects. Existence of non-additive effects may bias the estimation of the additive genetic variance. Model (8) was fitted to account for any full-sib effect. This was done for both traits assuming a Weibull baseline distribution. In such a case, when a log-gamma distribution is chosen for the full-sib effects, these effects can be algebraically integrated out. Unfortunately, all attempts to fit model (8) led to estimates of $\gamma$ tending towards $+\infty$, for LPL as for LRL. This is tantamount to a zero variance of the full-sib effect. Two interpretations are possible: either the correct genetic model is truly additive (there is no full-sib effect) or the amount of censoring was simply too large to allow a proper estimation of the $\gamma$ parameter. In order to roughly assess the influence of the full-sib effect on the estimated amount of additive genetic variation, model (8) was fitted again, arbitrarily assuming that the variance of the full-sib effect was equal to half the additive genetic variance initially obtained with model (6). As a result, the estimated additive genetic variances (Tab. I) were reduced by only $17 \%$ and $12 \%$ for LRL and LPL, respectively: not accounting for non-additive full-sib variance (if such a variance exists) led to a relatively modest overestimation of $\sigma_{g}^{2}$. 


\subsection{Genetic evaluation, environmental effects and genetic trend}

Using the modal estimates of the variance components, estimated sire and dam effects were obtained with each model indicated in Table I.

Raw correlations between these breeding values are indicated in Table II: for the same trait (LRL or LPL), correlations were very high $(>0.994)$ whatever the pair of models considered. These correlations were even higher for males $(>0.997)$ and within flock $(>0.998)$. On the other hand, correlations between LRL and LPL sire and dam effects were close to 0 .

Table II. Correlations between breeding values obtained with different models.

\begin{tabular}{lcccc}
\hline $\begin{array}{l}\text { Trait } \\
\text { Model }\end{array}$ & $\begin{array}{c}\mathrm{LRL}^{a} \\
\mathrm{Cox}^{b}\end{array}$ & Weibull $^{c}$ & + full-sib $^{d, g}$ & $\begin{array}{c}\text { LPL }^{a} \\
\text { Weibull }^{c}\end{array}$ \\
\hline LRL Weibull & 0.997 & & & \\
$\quad$ Weibull + full-sib & 0.994 & 0.997 & & \\
LPL Weibull & 0.049 & 0.039 & 0.042 & \\
$\quad$ Weibull + full-sib
\end{tabular}

$a, b, c, d, g$ as in Table I.

Hatch within flock (HWF) solutions varied widely, but without any particular trend. For LRL, the standard deviation of these solutions was 0.53 with extreme solutions at -1.73 and +1.68 : the hazard ratio between extreme hatches was equal to $\exp \{1.68-(-1.73)\}=30.3$. Some hatches had very low mortality rates. The corresponding values for LPL were equal to 0.45 for the standard deviation, -1.20 and +1.24 for the extreme solutions and 11.5 for the risk ratio between extreme hatches. The correlation between LRL and LPL HWF effects was equal to -0.095 and was not significantly different from 0 .

Estimated sire and dam effects were transformed into breeding values expressed in genetic standard deviations. Mean values per flock were very similar for males and females. With the use of 'correct' models (stratified Cox model for LRL, Weibull model for LPL), the genetic trend, obtained by regressing these mean values on flock number, was estimated at -0.0106 and -0.0036 additive genetic standard deviation per flock. These favourable trends (negative values correspond to less mortality) were extremely modest. After 18 flocks, this represented a change of $0.14 \hat{\sigma}_{g}$ and $0.06 \hat{\sigma}_{g}$, respectively.

\subsection{Simulated selection}

Parents of animals in flock 20 (that is, parents in set $\mathcal{P}$ ) were evaluated using only information available when mating pairs were formed. Asymptotic standard errors were also computed. The reliabilities of the breeding values of their own sires and dams (in $\mathcal{G P}$ ) were on the average equal to 0.426 and 0.282 for LRL, 0.360 and 0.247 for LPL. Therefore, assuming independence of parental breeding values, the reliabilities of pedigree values for the animals in $\mathcal{P}$ were equal to 0.177 for LRL and 0.152 for LPL. 


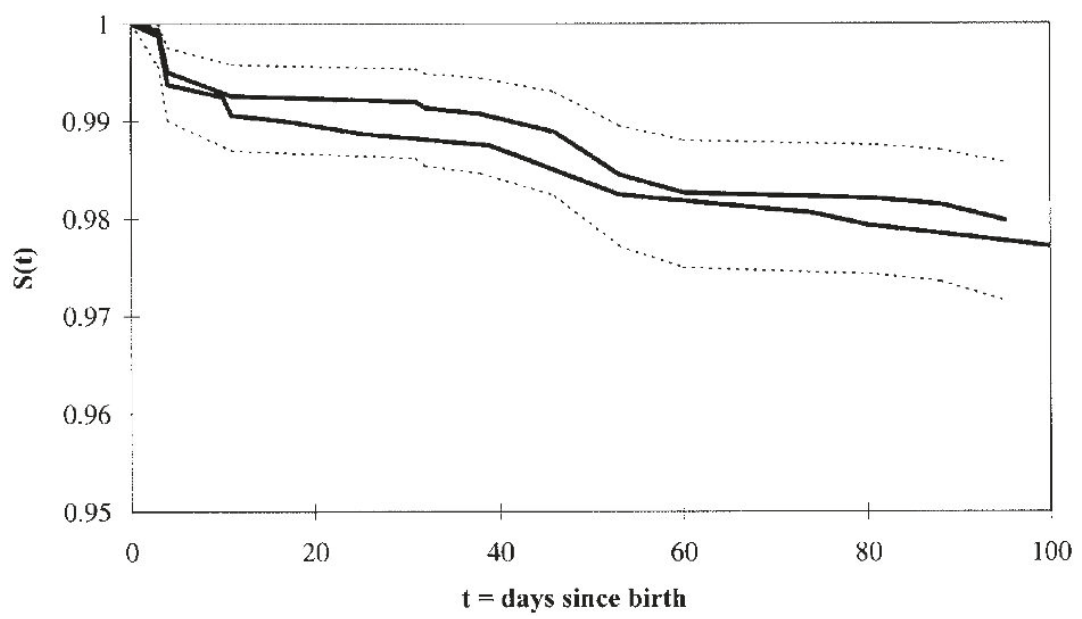

Figure 5. Kaplan-Meier non-parametric estimate of the survival curve of two extreme groups of animals, based on their pedigree value for survival during the rearing period (bottom solid line: worst group; top solid line: best group; dotted lines: $95 \%$ confidence interval for the best group).

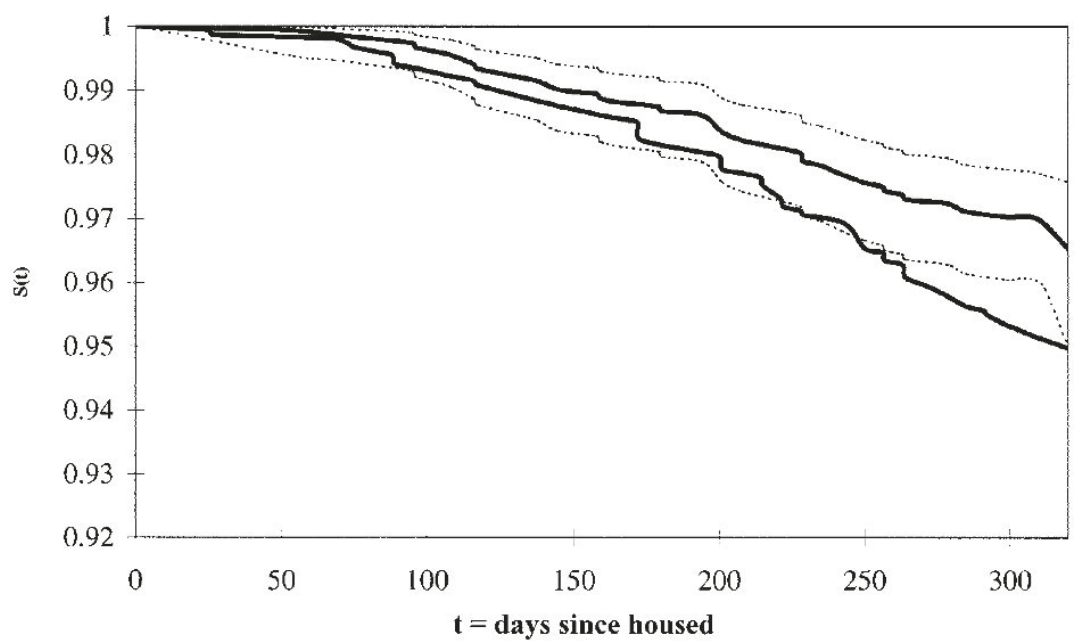

Figure 6. Kaplan-Meier non-parametric estimate of the survival curve of two extreme groups of animals, based on their pedigree value for survival during the production period (bottom solid line: worst group; top solid line: best group; dotted lines: $95 \%$ confidence interval for the best group).

Separately for each trait, records of animals in flock 20 were distributed into four groups, based on the sum of the pedigree values of their parents. Figures 5 and 6 represent the Kaplan-Meier estimate of the survivor curves of the top and bottom groups. The other two groups gave intermediate results.

For LRL, the difference between the two curves was small but consistent with the groups ranking: after about 100 days, $98.0 \%$ of the animals in the best group were still alive. For the worst group, the survival rate was $0.3 \%$ 
lower. However, this difference was much smaller than the confidence interval for each curve. It was also much smaller than the expected one. Using a formula similar to (9), a difference of $1.6 \%$ was anticipated. One interpretation is that the average hatch with flock effect for flock 20 was somewhat inflated by an abnormal mortality rate during the first week after birth. When the starting point was at day 7 , a difference of $0.6 \%$ in survival was predicted at 100 days of age, closer to the observed $0.3 \%$. The use of the actual estimate of the baseline hazard function in (9) of the Cox model would certainly have given a better agreement between observed and expected curves than the Weibull model used as an approximation.

For LPL, the picture was much clearer: 300 days after being housed, $97.0 \%$ of the hens were still alive in the best group. This percentage went down to $95.3 \%(-1.7 \%)$ for the worst group and the $95 \%$ confidence interval of each nonparametric curve barely overlapped at that point. This gap between the two extreme groups was in good agreement with the expectation (1.5\% at 300 days; Fig. 7).

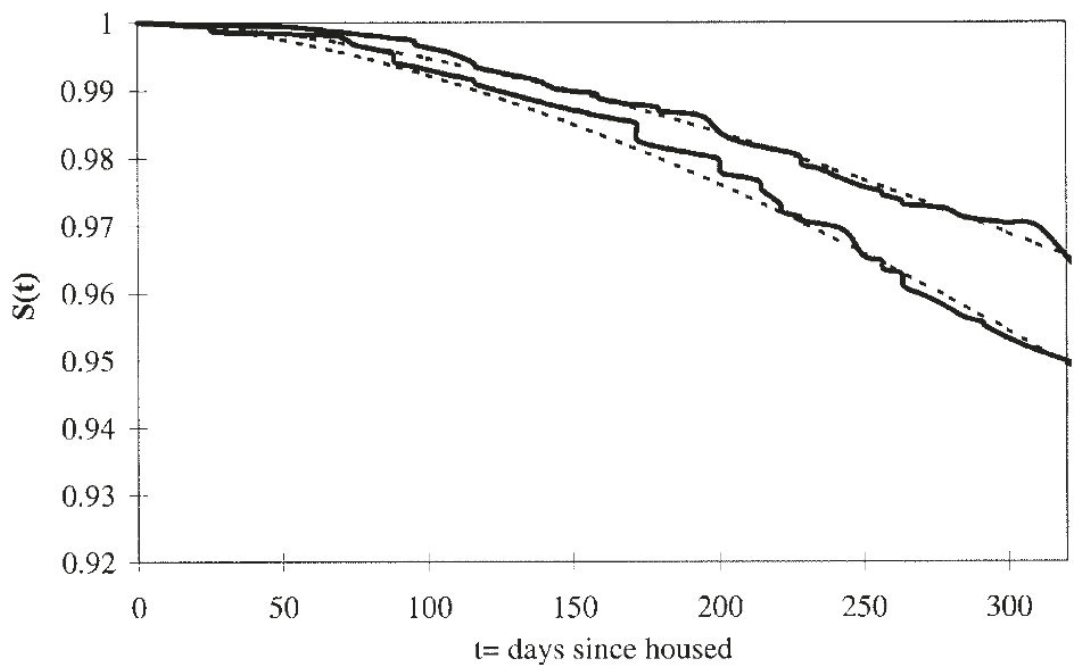

Figure 7. Observed (Kaplan-Meier) and predicted survival curves of two extreme groups of animals, during the production period (bottom solid line: worst group; top solid line: best group; dotted lines: predicted survival curves assuming the model (8) is correct).

\section{DISCUSSION}

\subsection{Traits and model selection}

This study clearly shows that LRL and LPL are completely different traits. The correlations between fixed effects or between genetic effects for both traits are virtually 0 . This is not really surprising since production is not interfering with maintenance and growth during the rearing period and there is 
no competition between animals when they stay in individual cages. Therefore, it appears fully justified to analyse LRL and LPL separately.

The economic importance of each trait is of course different: a key economic parameter for laying hens is the number of eggs laid per hen housed. Obviously, when a significant proportion of hens die during the production period, i.e., when LPL is deficient, it impairs the overall performance of the flock. LRL is probably not as essential: mortality during the rearing period only influences the production cost of young hens. However, it can be regarded as a good model for the study of survival in other production systems with many animals raised together in similar conditions and with a high censoring rate, as it is the case for broilers or turkeys. For most of the broiler breeders, liveability is a concern due to the economic loss, especially if mortality occurs as the birds approach market age. Presently, mortality is about 5\%. This rate may however increase due to the increasing incidence of ascites and sudden death syndrome, as continued selection pressure is maintained for increasing rates of growth [16].

It was hoped that a careful examination of baseline survival functions and of generalised residuals would permit a simple choice of a proper model of analysis. Unfortunately (but perhaps not surprisingly) graphical tests and the use of strongly correlated estimated residuals were clearly not powerful enough to put forward a definite 'best model'. Other techniques should be used (crossvalidation, use of overall goodness-of-fit tests, etc) but in survival analysis, censoring and random effects make their development more difficult. Still, several partial conclusions may be drawn: here, our analyses did not show any indication of a strong inadequacy of the proportional hazard model. For LPL, a straightforward Weibull model seemed to be adequate. For LRL, the baseline hazard functions of different flocks were not proportional and were not perfectly approximated by the Weibull hazard function. A stratified Cox model seemed more adequate but it was shown that genetic effects as well as estimated genetic parameters were almost identical, whether a Cox or Weibull model was used: once more, the Weibull frailty model appears very robust to (small?) departures of the baselines from the Weibull assumption. In practice, this is important because the Weibull model is computationally much simpler: the vector of first derivatives of the log-likelihood function is much easier to compute and the Hessian matrix is usually very sparse.

\subsection{Genetic parameters}

One of the most promising features of this study is the fact that LRL and LPL additive genetic variances could be estimated precisely, despite an extremely high censoring rate. The two traits were heritable and the particular pedigree structure - (almost) nested design, reasonably balanced family size, large number of progeny per sire - permitted to reveal important genetic differences. In contrast, non-additive genetic or non-genetic (e.g., maternal) effects could not be evidenced. This may have been a direct consequence of the high censoring rate and the limited full-sib group size. But even when a substantial part of the variance was arbitrarily attributed to a full-sib effect, the additive genetic variances for LRL and LPL remained large and genetic trends and rankings were virtually unchanged. 


\subsection{Genetic evaluation and selection}

Selection of this strain of laying hens did not include any viability trait during the studied period. The absence of any clear genetic trend on LPL may illustrate the independence of fitness from production traits. Another interpretation is that only more fit animals were able to cope with more demanding production levels and selection on production traits indirectly prevented viability from deteriorating. The slight favourable trend for LRL may have resulted from natural selection (animals dying during the rearing period were obviously excluded from the breeding program) combined with some culling before housing of apparently unhealthy young hens.

Genetic evaluation using a straightforward Weibull model was fast and simple. If desired, a Weibull animal model can be implemented. Such an evaluation of the 100665 animals took about 17 min $30 \mathrm{~s}$ (with no calculation of standard errors). However, compared to the sire-dam model (6), the animal model did not have a real impact on ranking of candidates to selection. Obviously, these candidates had censored records: their own record contained very limited information and the correlation between their estimated breeding values from the animal model and from their parental average using the siredam model was equal to 0.996 ! In practice, the use of a simpler sire-dam model is therefore sufficient.

Simulating selection using retrospective data gave a striking illustration of the efficiency of the proposed model to detect genetically more viable animals during the production period: despite a very low overall value, mortality rate was still reduced by more than a third in the best selected group compared with the worst one (3.0\% vs. $4.7 \%)$. This reduction was in close agreement with what was expected given the estimates of the parameters obtained. The results for LRL were not as clear-cut, although the observed survival curve of the animals in the best selected group was consistently higher than in the worst one. Still, it is essential to remember that two features of this retrospective analysis reduced the applied selection differential on both traits, compared to real life situations: first, selection on survival was applied to animals already selected on production traits. In practice, simultaneous selection on an aggregate value combining breeding values for survival trait(s) and production traits would be more efficient and would considerably enlarge the number of candidates to selection. Second, mating pairs were already established when the simulated selection was applied. Therefore, some of the best males for survival traits may have been randomly mated to the worst females. Of course, selection of the best parents before the matings would have been more efficient.

\section{CONCLUSION}

This study demonstrates that survival mixed models can be efficiently used to genetically improve the viability of domestic animals, even in situations where the overall mortality rate is very low. This seems to be particularly the case in poultry, for which data collection is relatively easy, pedigree structure is favourable, environmental factors are under strict control and contemporary groups are large. Furthermore, the implementation of a routine genetic evaluation based on survival models is clearly not computationally 
difficult, even on large populations. The inclusion of the results of such an evaluation in breeding programs seems possible and is probably advisable, for economic as well as for welfare reasons.

\section{REFERENCES}

[1] Bierdermann G., Petersen A., Lange K., Analysis of the results of random sample tests for laying hens in Hessia during thirty years, Archiv für Geflügelkunde 61 (1997) 97-105.

[2] Clayton D.G., Cuzick J., Multivariate generalizations of the proportional hazards model, J. Royal Stat. Soc., Series A 148 (1985) 82-117.

[3] Cox D.R., Regression models and life tables (with discussion), J. Royal Stat. Soc., Series B 34 (1972) 187-220.

[4] Cox D.R., Snell E., A general definition of residuals (with discussion), J. Royal Stat. Soc. Ser. B 30 (1968) 248-275.

[5] Cox D.R., Oakes D., Analysis of survival data, Chapman and Hall, London, UK, 1984.

[6] Ducrocq V., An analysis of length of productive life in dairy cattle, Ph.D. dissertation, Cornell University, Ithaca, NY, USA, 1987.

[7] Ducrocq V., Two years of experience with the French genetic evaluation of dairy bulls on production adjusted longevity of their daughters, in: Proceedings of the GIFT (E.U. Concerted Action on Genetic Improvement of Functional Traits) workshop on Longevity, Jouy-en-Josas, France; Interbull Bulletin, Uppsala, Sweden, 21 (1999) 60-67.

[8] Ducrocq V., Casella G., A Bayesian analysis of mixed survival models, Genet. Sel. Evol. 28 (1996) 505-529.

[9] Ducrocq V., Quaas R.L., Pollak E., Casella G., Length of productive life of dairy cows. I. Justification of a Weibull model, J. Dairy Sci. 71 (1988) 3061-3070.

[10] Ducrocq V., Quaas R.L., Pollak E., Casella G., Length of productive life of dairy cows. II. Variance component estimation and sire evaluation, J. Dairy Sci. 71 (1988) 3071-3079.

[11] Ducrocq V., Sölkner J., Implementation of a routine breeding value evaluation for longevity of dairy cows using survival analysis techniques, in: 6th World Congress on Genetics Applied to Livestock Production, 23 (1998) 359-362; Anim. Genetics and Breeding Unit, Univ. of New England, Armidale, Australia.

[12] Ducrocq V., Sölkner J., "The Survival Kit - V3.0", a package for large analyses of survival data, in: 6th World Congress on Genetics Applied to Livestock Production, 27 (1998) 447-448; Anim. Genetics and Breeding Unit, Univ. of New England, Armidale, Australia.

[13] Flock D.K., Genetic and non-genetic factors determining the success of eggtype breeding programs, in: Proceedings of 20th WPSA Congress, New Delhi, India, 1 (1996) 425-431.

[14] Foulley J.L., Prediction of selection response for threshold dichotomous traits, Genetics 132 (1992) 1187-1194.

[15] Foulley J.L., Prediction of selection response for Poisson distributed traits, Genet. Sel. Evol. 25 (1993) 297-303.

[16] Hunton P., Applied breeding and selection. Industrial breeding and selection, in: Crawford R.D. (Eds.), Poultry Breeding and Genetics, Chap. 40, 1990, 1123 pp.

[17] Kalbfleisch J.D., Prentice R.L., The statistical analysis of failure time data, John Wiley and sons, New-York, USA, 1980.

[18] Kaplan E.L., Meier P., Nonparametric estimation from incomplete observations, J. Amer. Stat. Ass. 53 (1958) 457-481. 
[19] Klein J.P., Semiparametric estimation of random effects using the Cox model based on the EM algorithm, Biometrics 48 (1992) 795-806.

[20] Klein J., Moeschberger M., Survival analysis, John Willey and sons, NewYork, USA, 1997.

[21] Preisinger R., Internationalisation of breeding programmes - breeding eggtype chickens for a global market, in: 6th World Congress on Genetics Applied to Livestock Production, 26 (1998) 135-142; Anim. Genetics and Breeding Unit, Univ. of New England, Armidale, Australia.

[22] Vaupel J., Manton K.G., Stallard E., The impact of heterogeneity in individual frailty and the dynamics of mortality, Demography 16 (1979) 439-454.

[23] Wei M., van der Werf J.H.J., Animal model estimation of additive and dominance variances in egg production traits in Poultry, J. Anim. Sci. 71 (1993) $57-65$. 